\title{
Observation of High-Energy Astrophysical Neutrinos in Three Years of IceCube Data
}

M. G. Aartsen, ${ }^{2}$ M. Ackermann, ${ }^{45}$ J. Adams, ${ }^{15}$ J. A. Aguilar, ${ }^{23}$ M. Ahlers,${ }^{28}$ M. Ahrens, ${ }^{36}$ D. Altmann,${ }^{22}$ T. Anderson,${ }^{42}$ C. Arguelles, ${ }^{28}$ T. C. Arlen, ${ }^{42}$ J. Auffenberg, ${ }^{1}$ X. Bai, ${ }^{34}$ S. W. Barwick, ${ }^{25}$ V. Baum, ${ }^{29}$ J. J. Beatty, ${ }^{17,18}$ J. Becker Tjus ${ }^{10}$ K.-H. Becker, ${ }^{44}$ S. BenZvi, ${ }^{28}$ P. Berghaus, ${ }^{45}$ D. Berley, ${ }^{16}$ E. Bernardini ${ }^{45}$ A. Bernhard,${ }^{31}$ D. Z. Besson, ${ }^{26}$ G. Binder, ${ }^{8,7}$ D. Bindig, ${ }^{44}$ M. Bissok, ${ }^{1}$ E. Blaufuss, ${ }^{16}$ J. Blumenthal, ${ }^{1}$ D. J. Boersma, ${ }^{43}$ C. Bohm, ${ }^{36}$ D. Bose, ${ }^{38}$ S. Böser, ${ }^{11}$ O. Botner, ${ }^{43}$ L. Brayeur, ${ }^{13}$ H.-P. Bretz, ${ }^{45}$ A. M. Brown, ${ }^{15}$ J. Casey, ${ }^{5}$ M. Casier, ${ }^{13}$ D. Chirkin, ${ }^{28}$ A. Christov, ${ }^{23}$ B. Christy, ${ }^{16}$ K. Clark, ${ }^{39}$ L. Classen, ${ }^{22}$ F. Clevermann, ${ }^{20}$ S. Coenders, ${ }^{31}$ D. F. Cowen, ${ }^{42,41}$ A. H. Cruz Silva, ${ }^{45}$ M. Danninger, ${ }^{36}$ J. Daughhetee, ${ }^{5}$ J. C. Davis, ${ }^{17}$ M. Day, ${ }^{28}$ J. P. A. M. de André, ${ }^{42}$ C. De Clercq, ${ }^{13}$ S. De Ridder, ${ }^{24}$ P. Desiati, ${ }^{28}$ K. D. de Vries, ${ }^{13}$ M. de With, ${ }^{9}$ T. DeYoung, ${ }^{42}$ J. C. Díaz-Vélez, ${ }^{28}$ M. Dunkman, ${ }^{42}$ R. Eagan, ${ }^{42}$ B. Eberhardt, ${ }^{29}$ B. Eichmann, ${ }^{10}$ J. Eisch, ${ }^{28}$ S. Euler, ${ }^{43}$ P. A. Evenson, ${ }^{32}$ O. Fadiran, ${ }^{28}$ A. R. Fazely, ${ }^{6}$ A. Fedynitch, ${ }^{10}$ J. Feintzeig,,${ }^{28,}{ }^{*}$ J. Felde, ${ }^{16}$ T. Feusels,${ }^{24}$ K. Filimonov, ${ }^{7}$ C. Finley, ${ }^{36}$ T. Fischer-Wasels, ${ }^{44}$ S. Flis, ${ }^{36}$ A. Franckowiak, ${ }^{11}$ K. Frantzen, ${ }^{20}$ T. Fuchs,${ }^{20}$ T. K. Gaisser, ${ }^{32}$ J. Gallagher, ${ }^{27}$ L. Gerhardt, ${ }^{8,7}$ D. Gier, ${ }^{1}$ L. Gladstone, ${ }^{28}$ T. Glüsenkamp, ${ }^{45}$ A. Goldschmidt, ${ }^{8}$ G. Golup, ${ }^{13}$ J. G. Gonzalez,${ }^{32}$ J. A. Goodman, ${ }^{16}$ D. Góra, ${ }^{45}$ D. T. Grandmont, ${ }^{21}$ D. Grant, ${ }^{21}$ P. Gretskov, ${ }^{1}$ J. C. Groh, ${ }^{42}$ A. Groß,${ }^{31}$ C. Ha, ${ }^{8,7}$ C. Haack, ${ }^{1}$ A. Haj Ismail, ${ }^{24}$ P. Hallen, ${ }^{1}$ A. Hallgren, ${ }^{43}$ F. Halzen, ${ }^{28}$ K. Hanson, ${ }^{12}$ D. Hebecker, ${ }^{11}$ D. Heereman, ${ }^{12}$ D. Heinen, ${ }^{1}$ K. Helbing, ${ }^{44}$ R. Hellauer, ${ }^{16}$ D. Hellwig, ${ }^{1}$ S. Hickford, ${ }^{15}$ G. C. Hill, ${ }^{2}$ K. D. Hoffman, ${ }^{16}$ R. Hoffmann, ${ }^{44}$ A. Homeier, ${ }^{11}$ K. Hoshina, ${ }^{28, \dagger}$ F. Huang ${ }^{42}$ W. Huelsnitz, ${ }^{16}$ P. O. Hulth, ${ }^{36}$ K. Hultqvist, ${ }^{36}$ S. Hussain, ${ }^{32}$ A. Ishihara, ${ }^{14}$ E. Jacobi, ${ }^{45}$ J. Jacobsen, ${ }^{28}$ K. Jagielski, ${ }^{1}$ G. S. Japaridze, ${ }^{4}$ K. Jero,${ }^{28}$ O. Jlelati, ${ }^{24}$ M. Jurkovic, ${ }^{31}$ B. Kaminsky, ${ }^{45}$ A. Kappes, ${ }^{22}$ T. Karg, ${ }^{45}$ A. Karle, ${ }^{28}$ M. Kauer, ${ }^{28}$ J. L. Kelley, ${ }^{28}$ A. Kheirandish, ${ }^{28}$ J. Kiryluk, ${ }^{37}$ J. Kläs, ${ }^{44}$ S. R. Klein, ${ }^{8,7}$ J.-H. Köhne, ${ }^{20}$ G. Kohnen, ${ }^{30}$ H. Kolanoski, ${ }^{9}$ A. Koob, ${ }^{1}$ L. Köpke, ${ }^{29}$ C. Kopper, ${ }^{28,}{ }^{*}$ S. Kopper, ${ }^{44}$ D. J. Koskinen, ${ }^{19}$ M. Kowalski, ${ }^{11}$ A. Kriesten, ${ }^{1}$ K. Krings, ${ }^{1}$ G. Kroll,${ }^{29}$ J. Kunnen, ${ }^{13}$ N. Kurahashi, ${ }^{28}$ T. Kuwabara, ${ }^{32}$ M. Labare, ${ }^{24}$ D. T. Larsen, ${ }^{28}$ M. J. Larson, ${ }^{19}$ M. Lesiak-Bzdak, ${ }^{37}$ M. Leuermann, ${ }^{1}$ J. Leute, ${ }^{31}$ J. Lünemann, ${ }^{29}$ O. Macías, ${ }^{15}$ J. Madsen,${ }^{35}$ G. Maggi, ${ }^{13}$ R. Maruyama, ${ }^{28}$ K. Mase, ${ }^{14}$ H. S. Matis, ${ }^{8}$ F. McNally, ${ }^{28}$ K. Meagher, ${ }^{16}$ A. Meli, ${ }^{24}$ T. Meures, ${ }^{12}$ S. Miarecki ${ }^{8,7}$ E. Middell ${ }^{45}$ E. Middlemas, ${ }^{28}$ N. Milke,${ }^{20}$ J. Miller,${ }^{13}$ L. Mohrmann, ${ }^{45}$ T. Montaruli, ${ }^{23}$ R. Morse, ${ }^{28}$ R. Nahnhauer, ${ }^{45}$ U. Naumann, ${ }^{44}$ H. Niederhausen, ${ }^{37}$ S. C. Nowicki, ${ }^{21}$

D. R. Nygren, ${ }^{8}$ A. Obertacke, ${ }^{44}$ S. Odrowski, ${ }^{21}$ A. Olivas, ${ }^{16}$ A. Omairat, ${ }^{44}$ A. O'Murchadha, ${ }^{12}$ T. Palczewski, ${ }^{40}$ L. Paul, ${ }^{1}$ Ö. Penek, ${ }^{1}$ J. A. Pepper ${ }^{40}$ C. Pérez de los Heros,${ }^{43}$ C. Pfendner,${ }^{17}$ D. Pieloth,${ }^{20}$ E. Pinat, ${ }^{12}$ J. Posselt,${ }^{44}$ P. B. Price, ${ }^{7}$ G. T. Przybylski, ${ }^{8}$ J. Pütz, ${ }^{1}$ M. Quinnan, ${ }^{42}$ L. Rädel, ${ }^{1}$ M. Rameez,${ }^{23}$ K. Rawlins,${ }^{3}$ P. Redl, ${ }^{16}$ I. Rees,${ }^{28}$ R. Reimann, ${ }^{1}$ E. Resconi ${ }^{31}$ W. Rhode, ${ }^{20}$ M. Richman, ${ }^{16}$ B. Riedel, ${ }^{28}$ S. Robertson, ${ }^{2}$ J. P. Rodrigues, ${ }^{28}$ M. Rongen, ${ }^{1}$ C. Rott, ${ }^{38}$ T. Ruhe,${ }^{20}$ B. Ruzybayev, ${ }^{32}$ D. Ryckbosch, ${ }^{24}$ S. M. Saba, ${ }^{10}$ H.-G. Sander, ${ }^{29}$ M. Santander ${ }^{28}$ S. Sarkar,${ }^{19,33}$ K. Schatto,${ }^{29}$ F. Scheriau, ${ }^{20}$ T. Schmidt, ${ }^{16}$ M. Schmitz, ${ }^{20}$ S. Schoenen, ${ }^{1}$ S. Schöneberg, ${ }^{10}$ A. Schönwald, ${ }^{45}$ A. Schukraft,,${ }^{1}$ L. Schulte, ${ }^{11}$ O. Schulz, ${ }^{31}$ D. Seckel,${ }^{32}$ Y. Sestayo, ${ }^{31}$ S. Seunarine,${ }^{35}$ R. Shanidze, ${ }^{45}$ C. Sheremata, ${ }^{21}$ M. W. E. Smith ${ }^{42}$ D. Soldin, ${ }^{44}$ G. M. Spiczak,${ }^{35}$ C. Spiering, ${ }^{45}$ M. Stamatikos, ${ }^{17,}$ T. Stanev, ${ }^{32}$ N. A. Stanisha, ${ }^{42}$ A. Stasik, ${ }^{11}$ T. Stezelberger, ${ }^{8}$ R. G. Stokstad ${ }^{8}$ A. Stöß1, E. A. Strahler, ${ }^{13}$ R. Ström, ${ }^{43}$ N. L. Strotjohann, ${ }^{11}$ G. W. Sullivan, ${ }^{16}$ H. Taavola ${ }^{43}$ I. Taboada, ${ }^{5}$ A. Tamburro, ${ }^{32}$ A. Tepe,${ }^{44}$ S. Ter-Antonyan, ${ }^{6}$ A. Terliuk, ${ }^{45}$ G. Tešić, ${ }^{42}$ S. Tilav, ${ }^{32}$ P. A. Toale, ${ }^{40}$ M. N. Tobin,${ }^{28}$ D. Tosi, ${ }^{28}$ M. Tselengidou, ${ }^{22}$ E. Unger, ${ }^{10}$

M. Usner, ${ }^{11}$ S. Vallecorsa, ${ }^{23}$ N. van Eijndhoven, ${ }^{13}$ J. Vandenbroucke ${ }^{28}$ J. van Santen, ${ }^{28}$ M. Vehring, ${ }^{1}$ M. Voge, ${ }^{11}$

M. Vraeghe, ${ }^{24} \mathrm{C}$. Walck, ${ }^{36} \mathrm{M}$. Wallraff, ${ }^{1} \mathrm{Ch}$. Weaver, ${ }^{28} \mathrm{M}$. Wellons ${ }^{28} \mathrm{C}$. Wendt, ${ }^{28} \mathrm{~S}$. Westerhoff, ${ }^{28} \mathrm{~B}$. J. Whelan, ${ }^{2}$ N. Whitehorn, ${ }^{28,7, *}$ C. Wichary, ${ }^{1}$ K. Wiebe, ${ }^{29}$ C. H. Wiebusch, ${ }^{1}$ D. R. Williams, ${ }^{40}$ H. Wissing, ${ }^{16}$ M. Wolf, ${ }^{36}$ T. R. Wood, ${ }^{21}$ K. Woschnagg, ${ }^{7}$ D. L. Xu, ${ }^{40}$ X. W. Xu, ${ }^{6}$ J. P. Yanez, ${ }^{45}$ G. Yodh, ${ }^{25}$ S. Yoshida, ${ }^{14}$ P. Zarzhitsky, ${ }^{40}$ J. Ziemann, ${ }^{20}$ S. Zierke, ${ }^{1}$ and M. Zoll ${ }^{36}$

(IceCube Collaboration)

${ }^{1}$ III. Physikalisches Institut, RWTH Aachen University, D-52056 Aachen, Germany

${ }^{2}$ School of Chemistry \& Physics, University of Adelaide, Adelaide, South Australia 5005 Australia

${ }^{3}$ Department of Physics and Astronomy, University of Alaska Anchorage, 3211 Providence Drive, Anchorage, Alaska 99508, USA ${ }^{4}$ CTSPS, Clark-Atlanta University, Atlanta, Georgia 30314, USA

${ }^{5}$ School of Physics and Center for Relativistic Astrophysics, Georgia Institute of Technology, Atlanta, Georgia 30332, USA

${ }^{6}$ Department of Physics, Southern University, Baton Rouge, Louisiana 70813, USA

${ }^{7}$ Department of Physics, University of California, Berkeley, California 94720, USA

${ }^{8}$ Lawrence Berkeley National Laboratory, Berkeley, California 94720, USA

${ }^{9}$ Institut für Physik, Humboldt-Universität zu Berlin, D-12489 Berlin, Germany

${ }^{10}$ Fakultät für Physik \& Astronomie, Ruhr-Universität Bochum, D-44780 Bochum, Germany 


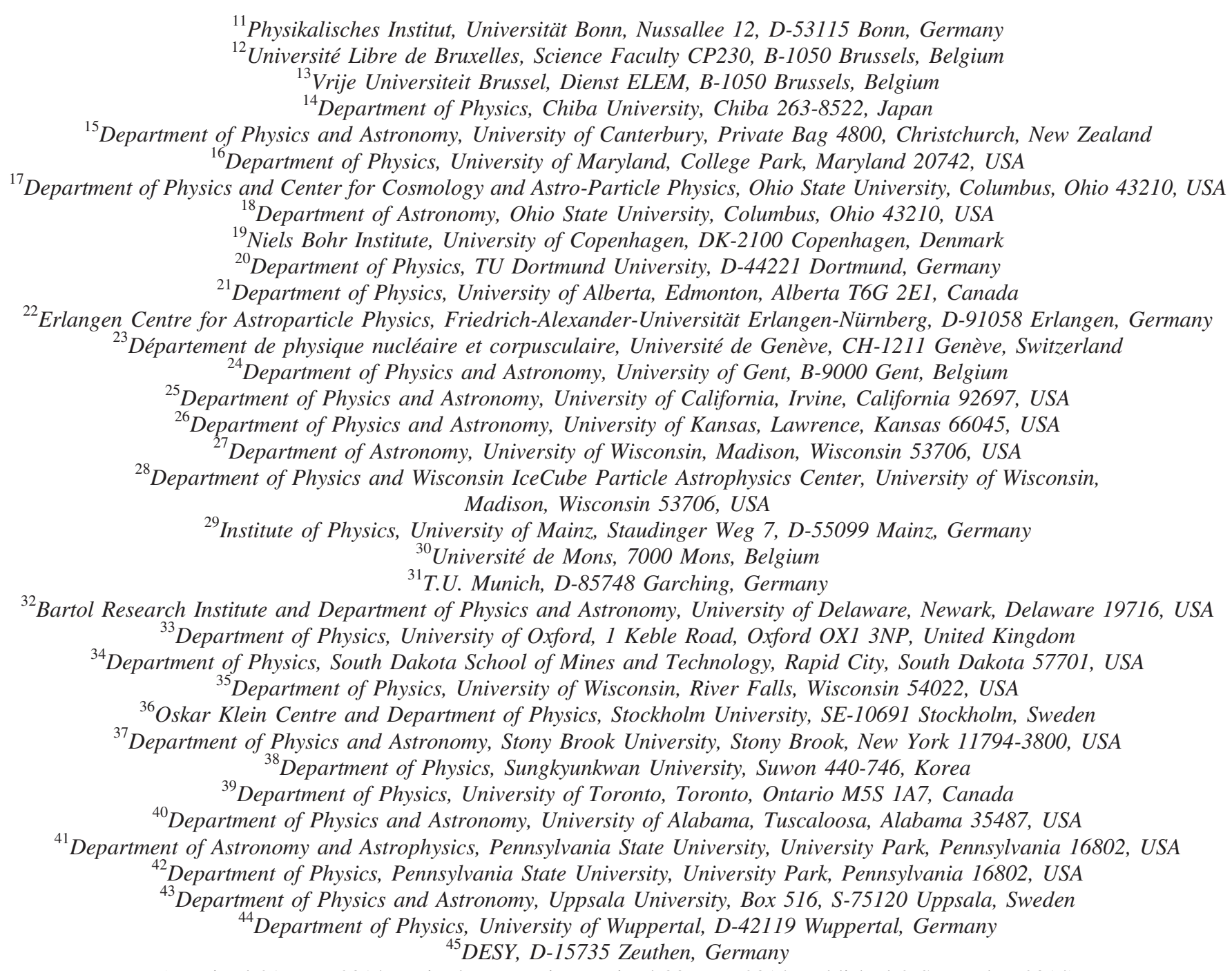

(Received 21 May 2014; revised manuscript received 30 June 2014; published 2 September 2014)

A search for high-energy neutrinos interacting within the IceCube detector between 2010 and 2012 provided the first evidence for a high-energy neutrino flux of extraterrestrial origin. Results from an analysis using the same methods with a third year (2012-2013) of data from the complete IceCube detector are consistent with the previously reported astrophysical flux in the $100 \mathrm{TeV}-\mathrm{PeV}$ range at the level of $10^{-8} \mathrm{GeV} \mathrm{cm}^{-2} \mathrm{~s}^{-1} \mathrm{sr}^{-1}$ per flavor and reject a purely atmospheric explanation for the combined three-year data at 5.7 $\sigma$. The data are consistent with expectations for equal fluxes of all three neutrino flavors and with isotropic arrival directions, suggesting either numerous or spatially extended sources. The three-year data set, with a live time of 988 days, contains a total of 37 neutrino candidate events with deposited energies ranging from 30 to $2000 \mathrm{TeV}$. The $2000-\mathrm{TeV}$ event is the highest-energy neutrino interaction ever observed.

High-energy neutrinos are expected to be produced in astrophysical objects by the decays of charged pions made in cosmic-ray interactions with radiation or gas [1-4]. As these pions decay, they produce neutrinos with typical energies of 5\% compared to those of the cosmic-ray nucleons $[5,6]$. These neutrinos can travel long distances undisturbed by either the absorption experienced by highenergy photons or the magnetic deflection experienced by charged particles, making them a unique tracer of cosmicray acceleration.

Observations since 2008 using the Antarctic gigaton IceCube detector [7] while it was under construction provided several indications of such neutrinos in a variety of channels [8-10]. Two years of data from the full detector, from May 2010 to May 2012, then provided the first strong evidence for the detection of these astrophysical neutrinos 
[11] using an all-flavor all-direction sample of neutrinos interacting within the detector volume. This analysis focused on neutrinos above $100 \mathrm{TeV}$, at which the expected atmospheric neutrino background falls to the level of one event per year, allowing any harder astrophysical flux to be seen clearly. Here, following the same techniques, we add a third year of data supporting this result and begin to probe the properties of the observed astrophysical neutrino flux.

Neutrinos are detected in IceCube by observing the Cherenkov light produced in ice by charged particles created when neutrinos interact. These particles generally travel distances too small to be resolved individually, and the particle shower is observed only in aggregate. In $\nu_{\mu}$ charged-current (CC) interactions, however, as well as a minority of $\nu_{\tau} \mathrm{CC}$, a high-energy muon is produced that leaves a visible track (unless produced on the detector boundary heading outward). Although deposited energy resolution is similar for all events, angular resolution for events that contain visible muon tracks is much better $\left(\lesssim 1^{\circ}, 50 \% \mathrm{CL}\right)$ than for those that do not $\left(\sim 15^{\circ}, 50 \% \mathrm{CL}\right)$ [12]. For equal neutrino fluxes of all flavors $(1: 1: 1), \nu_{\mu} \mathrm{CC}$ events make up only $20 \%$ of interactions [13].

Backgrounds to astrophysical neutrino detection arise entirely from cosmic-ray air showers. Muons produced by $\pi$ and $K$ decays above IceCube enter the detector at $2.8 \mathrm{kHz}$. Neutrinos produced in the same interactions [14-17] enter IceCube from above and below, and are seen at a much lower rate due to the low neutrino interaction cross section. Because $\pi$ and $K$ mesons decay overwhelmingly to muons rather than electrons, these neutrinos are predominantly $\nu_{\mu}$ and usually have tracktype topologies in the detector [13]. As the parent meson's energy rises, its lifetime increases, making it increasingly likely to interact before decaying. Both the atmospheric muon and neutrino fluxes thus become suppressed at high energy, with a spectrum one power steeper than the primary cosmic rays that produced them [18]. At energies above $\sim 100 \mathrm{TeV}$, an analogous flux of muons and neutrinos from the decay of charmed mesons is expected to dominate, as the shorter lifetime of these particles allows this flux to avoid suppression from interaction before decay [19-25]. This flux has not yet been observed, however, and both its overall rate and crossover energy with the $\pi / K$ flux are, at present, poorly constrained [26]. As before [11], we estimate all atmospheric neutrino background rates using measurements of the northern-hemisphere $\nu_{\mu}$ spectrum [9].

Event selection identifies neutrino interactions in IceCube by rejecting those events with Cherenkov-radiating particles, principally cosmic-ray muons, entering from outside the detector. As before, we used a simple anticoincidence muon veto in the outer layers of the detector [11], requiring that fewer than three of the first 250 detected photoelectrons (PE) be on the detector boundary. To ensure sufficient numbers of photons to reliably trigger this veto, we additionally required at least $6000 \mathrm{PE}$ overall, corresponding to deposited energies of approximately $30 \mathrm{TeV}$. This rejects all but one part in $10^{5}$ of the cosmic-ray muon background above $6000 \mathrm{PE}$ while providing a direction and topology-neutral neutrino sample [11]. We use a data-driven method to estimate this background by using one region of IceCube to tag muons and then measuring their detection rate in a separate layer of PMTs equivalent to our veto; this predicts a total muon background in three years of $8.4 \pm 4.2$ events. Rejection of events containing entering muons also significantly reduces downgoing atmospheric neutrinos (the southern hemisphere) by detecting and vetoing muons produced in the neutrinos' parent air showers [27,28]. This southernhemisphere suppression is a distinctive and generic feature of any neutrinos originating in cosmic-ray interactions in the atmosphere.

In the full 988-day sample, we detected 37 events (Fig. 1) with these characteristics relative to an expected background of $8.4 \pm 4.2$ cosmic-ray muon events and $6.6_{-1.6}^{+5.9}$ atmospheric neutrinos. Nine were observed in the third year. One of these (event 32) was produced by a coincident pair of background muons from unrelated air showers. This event cannot be reconstructed with a single direction and energy, and is excluded from the remainder of this article where these quantities are required. This event, like event 28, had subthreshold early hits in the IceTop surface array and our veto region, and is likely part of the expected muon background. Three additional downgoing track events are ambiguous; the remainder are uniformly distributed through the detector and appear to be neutrino interactions.

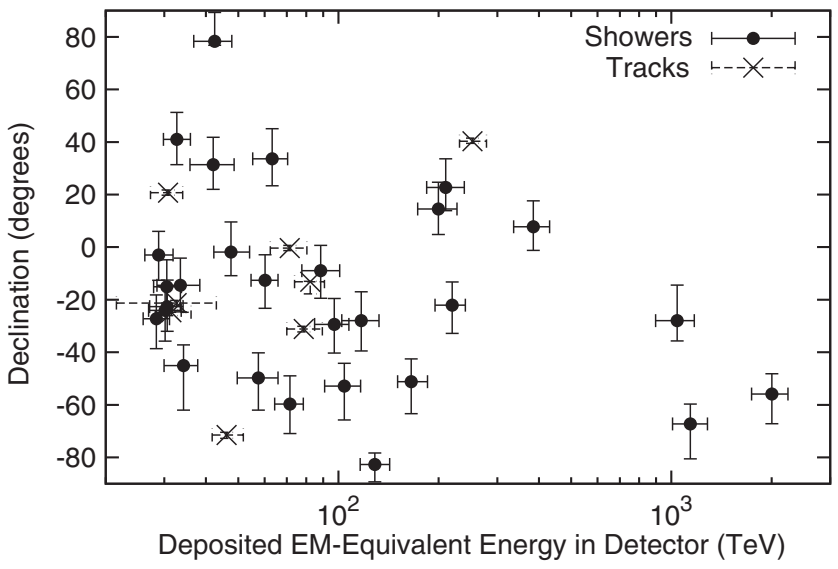

FIG. 1. Arrival angles and deposited energies of the events. Cosmic-ray muon background would appear as low-energy track events in the southern sky (bottom). Atmospheric neutrino backgrounds would appear primarily in the northern sky (top), also at low energies and predominantly as tracks. The attenuation of high-energy neutrinos in the Earth is visible in the top right of the figure. One event, a pair of coincident unrelated cosmic-ray muons, is excluded from this plot. A tabular version of these data, including additional information such as event times, can be found in Ref. [29]. 


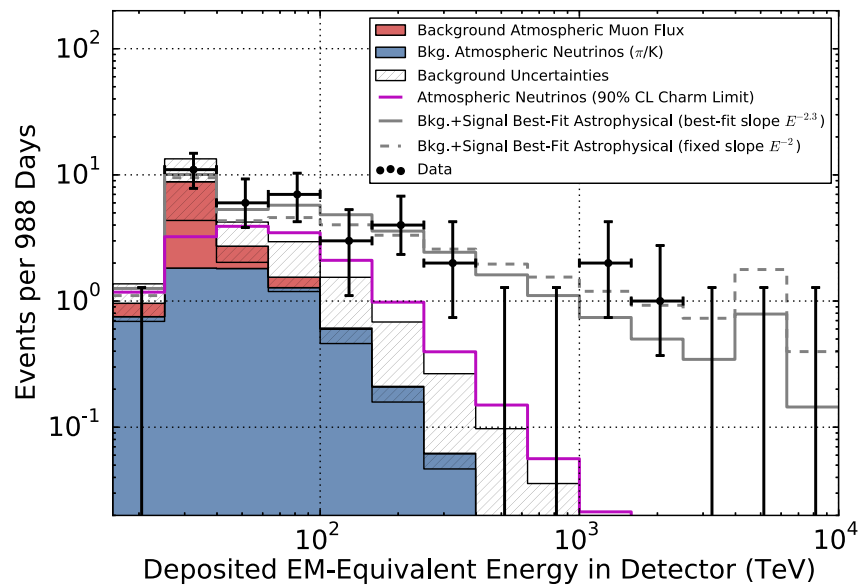

FIG. 2 (color online). Deposited energies of observed events with predictions. The hashed region shows uncertainties on the sum of all backgrounds. Muons (red) are computed from simulation to overcome statistical limitations in our background measurement and scaled to match the total measured background rate. Atmospheric neutrinos and uncertainties thereon are derived from previous measurements of both the $\pi / K$ and charm components of the atmospheric $\nu_{\mu}$ spectrum [9]. A gap larger than the one between 400 and $1000 \mathrm{TeV}$ appears in $43 \%$ of the realizations of the best-fit continuous spectrum.

A purely atmospheric explanation for these events is strongly disfavored by their properties. The observed deposited energy distribution extends to much higher energies (above $2 \mathrm{PeV}$, Fig. 2) than expected from the $\pi / K$ atmospheric neutrino background, which has been measured up to $100 \mathrm{TeV}$ [9]. While a harder spectrum is expected from atmospheric neutrinos produced in charmed meson decay, this possibility is constrained by the observed angular distribution. Although such neutrinos are produced isotropically, approximately half $[27,28]$ of those in the southern hemisphere are produced with muons of high enough energy to reach IceCube and trigger our muon veto. This results in a southern hemisphere charm rate $~ 50 \%$ smaller than the northern hemisphere rate, with larger ratios near the poles. Our data show no evidence of such a suppression, which is expected at some level from any atmospheric source of neutrinos (Fig. 3).

As in [11], we quantify these arguments using a likelihood fit in arrival angle and deposited energy to a combination of background muons, atmospheric neutrinos from $\pi / K$ decay, atmospheric neutrinos from charmed meson decay, and an isotropic 1:1:1 astrophysical $E^{-2}$ test flux, as expected from charged pion decays in cosmicray accelerators [30-33]. The fit included all events with $60 \mathrm{TeV}<E_{\text {dep }}<3 \mathrm{PeV}$. The expected muon background in this range is below 1 event in the three-year sample, minimizing imprecisions in modeling the muon background and threshold region. The normalizations of all background and signal neutrino fluxes were left free in the fit, without reference to uncertainties from [9], for maximal

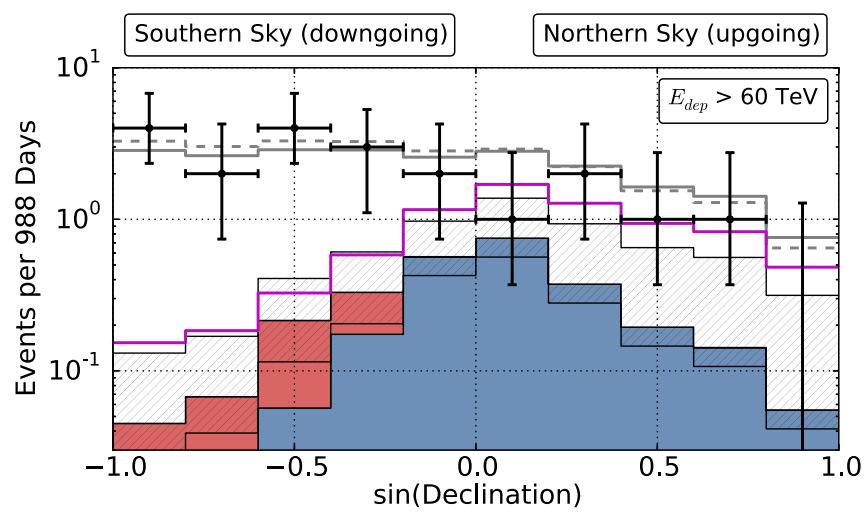

FIG. 3 (color online). Arrival angles of events with $E_{\text {dep }}>60 \mathrm{TeV}$, as used in our fit and above the majority of the cosmic-ray muon background. The increasing opacity of the Earth to high-energy neutrinos is visible on the right side of the plot. Vetoing atmospheric neutrinos by muons from their parent air showers depresses the atmospheric neutrino background on the left. The data are described well by the expected backgrounds and a hard astrophysical isotropic neutrino flux (gray lines). Colors are as in Fig. 2. Variations of this figure with other energy thresholds are in Ref. [29].

robustness. The penetrating muon background was constrained with a Gaussian prior reflecting our veto efficiency measurement. We obtain a best-fit per-flavor astrophysical flux $(\nu+\bar{\nu})$ in this energy range of $E^{2} \phi(E)=0.95 \pm 0.3 \times$ $10^{-8} \mathrm{GeV} \mathrm{cm}^{-2} \mathrm{~s}^{-1} \mathrm{sr}^{-1}$ and background normalizations within the expected ranges. Quoted errors are $1 \sigma$ uncertainties from a profile likelihood scan. This model describes the data well, with both the energy spectrum (Fig. 2) and arrival directions (Fig. 3) of the events consistent with expectations for an origin in a hard isotropic 1:1:1 neutrino flux. The best-fit atmospheric-only alternative model, however, would require a charm normalization 3.6 times higher than our current 90\% CL upper limit from the northern hemisphere $\nu_{\mu}$ spectrum [9]. Even this extreme scenario is disfavored by the energy and angular distributions of the events at $5.7 \sigma$ using a likelihood ratio test.

Figure 4 shows a fit using a more general model in which the astrophysical flux is parametrized as a piecewise function of energy rather than a continuous unbroken $E^{-2}$ power law. As before, we assume a 1:1:1 flavor ratio and isotropy. While the reconstructed spectrum is compatible with our earlier $E^{-2}$ ansatz, an unbroken $E^{-2}$ flux at our best-fit level predicts 3.1 additional events above $2 \mathrm{PeV}$ (a higher energy search [10] also saw none). This may indicate, along with the slight excess in lower energy bins, either a softer spectrum or a cutoff at high energies. Correlated systematic uncertainties in the first few points in the reconstructed spectrum (Fig. 4) arise from the poorly constrained level of the charm atmospheric neutrino background. The presence of this softer $\left(E^{-2.7}\right)$ component would decrease the nonatmospheric excess at low energies, 


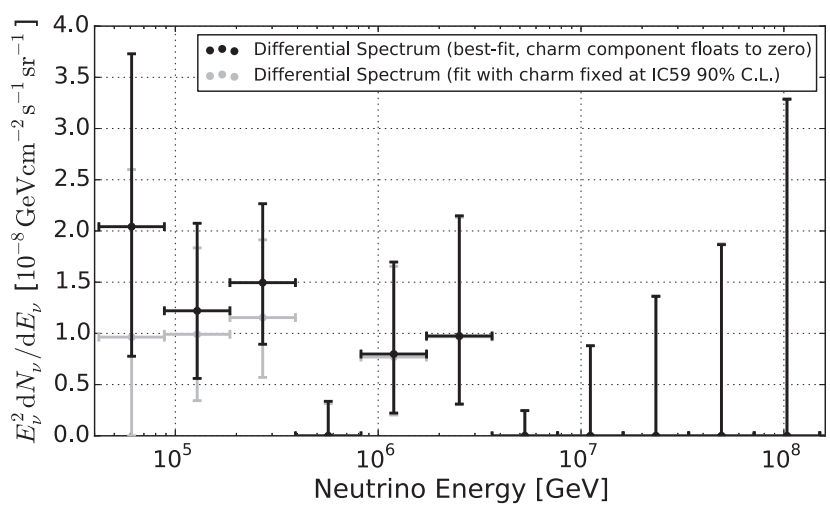

FIG. 4. Extraterrestrial neutrino flux $(\nu+\bar{\nu})$ as a function of energy. Vertical error bars indicate the $2 \Delta \mathcal{L}= \pm 1$ contours of the flux in each energy bin, holding all other values, including background normalizations, fixed. These provide approximate $68 \%$ confidence ranges. An increase in the charm atmospheric background to the level of the 90\% CL limit from the northern hemisphere $\nu_{\mu}$ spectrum [9] would reduce the inferred astrophysical flux at low energies to the level shown for comparison in light gray. The best-fit power law is $E^{2} \phi(E)=$ $1.5 \times 10^{-8}(E / 100 \mathrm{TeV})^{-0.3} \mathrm{GeV} \mathrm{cm}^{-2} \mathrm{~s}^{-1} \mathrm{sr}^{-1}$.

hardening the spectrum of the remaining data. The corresponding range of best-fit astrophysical slopes within our current $90 \%$ confidence band on the charm flux [9] is -2.0 to -2.3 . As the best-fit charm flux is zero, the best-fit astrophysical spectrum is on the lower boundary of this interval at -2.3 (solid line, Figs. 2 and 3) with a total statistical and systematic uncertainty of \pm 0.3 .

To identify any bright neutrino sources in the data, we employed the same maximum-likelihood clustering search as before [11] and searched for directional correlations with $\mathrm{TeV}$ gamma-ray sources. For all tests, the test statistic (TS) is defined as the logarithm of the ratio between the best-fit likelihood including a point source component and the likelihood for the null hypothesis, an isotropic distribution [34]. We determined the significance of any excess by comparing to maps scrambled in right ascension, in which our polar detector has uniform exposure.

As in [11], the clustering analysis was run twice, first with the entire event sample, after removing the two events (28 and 32) with strong evidence of a cosmic-ray origin, and second with only the 28 shower events. This controls for bias in the likelihood fit toward the positions of single well-resolved muon tracks. We also conducted an additional test in which we marginalize the likelihood over a uniform prior on the position of the hypothetical point source. This reduces the bias introduced by muons, allowing track and shower events to be used together, and it improves the sensitivity to multiple sources by considering the entire sky rather than the single best point.

Three tests were performed to search for neutrinos correlated with known gamma-ray sources, also using track and shower events together. The first two searched

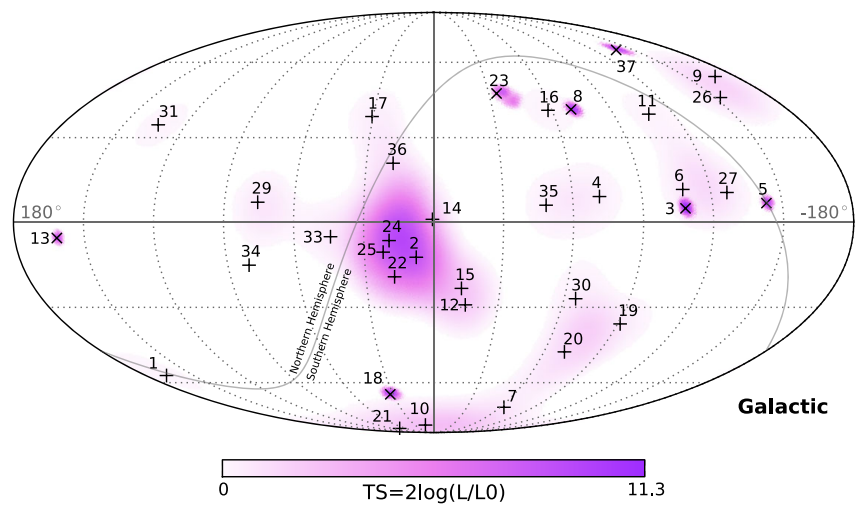

FIG. 5 (color online). Arrival directions of the events in Galactic coordinates. Showerlike events (median angular resolution $\sim 15^{\circ}$ ) are marked with + and those containing muon tracks $\left(\lesssim 1^{\circ}\right)$ with $\times$. Approximately $40 \%$ of the events (mostly tracks [13]) are expected to originate from atmospheric backgrounds. Event IDs match those in the catalog in Ref. [29] and are time ordered. The grey line denotes the equatorial plane. Colors show the test statistic (TS) for the point source clustering test at each location. No significant clustering was observed.

for clustering along the Galactic Plane, with a fixed width of $\pm 2.5^{\circ}$, based on $\mathrm{TeV}$ gamma-ray measurements [35], and with a free width between $\pm 2.5^{\circ}$ and $\pm 30^{\circ}$. The last test searched for the correlation between neutrino events and a predefined catalog of potential point sources (a combination of the usual IceCube [36] and ANTARES [37] lists; see Ref. [29]). For the catalog search, the TS value was evaluated at each source location, and the post-trial significance was calculated by comparing the highest observed value in each hemisphere to results from performing the analysis on scrambled data sets.

No hypothesis test yielded statistically significant evidence of clustering or correlations. For the all-sky clustering test (Fig. 5), scrambled data sets produced locations with equal or greater TS $84 \%$ and $7.2 \%$ of the time for all events and for showerlike events only. As in the two-year data set, the strongest clustering was near the Galactic Center. Other neutrino observations of this location give no evidence for a source [38], however, and no new events were strongly correlated with this region. When using the marginalized likelihood, a test statistic greater than or equal to the observed value was found in $28 \%$ of scrambled data sets. The source list yielded $p$ values for the northern and southern hemispheres of $28 \%$ and $8 \%$, respectively. Correlation with the Galactic Plane was not significant either: when letting the width float freely, the best fit was $\pm 7.5^{\circ}$ with a post-trials chance probability of $2.8 \%$, while a fixed width of $\pm 2.5^{\circ}$ returned a $p$ value of $24 \%$. A repeat of the time clustering search from [11] found no evidence of structure either.

With or without a possible Galactic contributions $[39,40]$, the high Galactic latitudes of many of the highestenergy events (Fig. 5) suggest at least some extragalactic 
component. Exceptions may be made for local large diffuse sources (e.g., the Fermi bubbles [41] or the Galactic Halo $[42,43])$, but these models can typically explain, at most, a fraction of the data. If our data arise from an extragalactic flux produced by many isotropically distributed point sources, we can compare our all-sky flux with existing point-source limits. By exploiting the additional effective volume provided by using uncontained $\nu_{\mu}$ events, previous point-source studies would have been sensitive to a northern sky point source producing more than 1\%-10\% of our best-fit flux, depending on the declination and energy spectrum [44]. The lack of any evidence for such sources from these studies, as well as the wide distribution of our events, thus lends support to an interpretation in terms of many individually dim sources. Some contribution from a few comparatively bright sources cannot be ruled out, however, especially in the southern hemisphere, where the sensitivity of IceCube to point sources in uncontained $\nu_{\mu}$ is reduced by the large muon background and small target mass above the detector.

The neutrino spectrum (Fig. 4) can also be used to constrain source properties. In almost all candidate sources [45-69], neutrinos would be produced by the interaction of cosmic rays with either radiation or gas. Interactions with radiation $(p \gamma)$ typically produce a peaked spectrum, reflecting the energy spectrum of the photons; those with gas $(p p)$ produce a smooth power law $[5,6]$. While $p \gamma$ models satisfactorily explain some aspects of the data such as the possible dropoff at high energies, many involve a central plateau smaller than our observed energy range, placing them in weak tension with the data. As an example, the $p \gamma$ AGN spectrum in [45] peaks at several $\mathrm{PeV}$ with much lower predictions at $100 \mathrm{TeV}$; thus, while able to explain the highest energy events, it fits poorly at lower energies and is disfavored as the sole source at the $2 \sigma$ level with respect to our simple $E^{-2}$ test flux. Gamma-ray burst $p \gamma$ models such as in [58,59] have energy ranges better aligned with our data, with central plateaus from around $100 \mathrm{TeV}$ to a few $\mathrm{PeV}$, although existing limits from searches for correlations with observed GRBs are more than an order of magnitude below the observed flux [70]. Cosmic-ray interactions with gas, such as predicted around supernova remnants in our and other galaxies, particularly those with high star-forming rates, produce smooth spectra with slopes reflecting post-diffusion cosmic rays (e.g., $E^{-2.2}$ in [66]) and seem to describe the data well. Large uncertainties on both the measured neutrino spectrum and all models prevent any conclusions, however.

The best-fit flux level in our central energy range $\left(10^{-8} \mathrm{GeV} \mathrm{cm}^{-2} \mathrm{~s}^{-1} \mathrm{sr}^{-1}\right.$ per flavor) is similar to the Waxman-Bahcall bound [71], the aggregate neutrino flux from charged pion decay in all extragalactic cosmic-ray accelerators if they are optically thin. This bound is derived from the cosmic-ray spectrum above $10^{18} \mathrm{eV}(1000 \mathrm{PeV})$. Our neutrinos, however, are likely associated with protons at much lower energies, on the order of 1 to $10 \mathrm{PeV}[5,6]$, at which the bound may be quite different [72]. Along with large uncertainties in the neutrino spectrum (Fig. 4), this makes correspondence with the Waxman-Bahcall bound, or $10^{18} \mathrm{eV}$ cosmic-ray sources, unclear.

Further observations with the present or upgraded IceCube detector and the planned KM3NeT [73] telescope are required to answer many questions about the sources of this astrophysical flux [74]. Gamma-ray, optical, and x-ray observations of the directions of individual high-energy neutrinos, which point directly to their origins, may also be able to identify these sources even for those with neutrino luminosities too low for identification from neutrino measurements alone.

We acknowledge support from the following agencies: U.S. National Science Foundation-Office of Polar Programs, U.S. National Science Foundation-Physics Division, University of Wisconsin Alumni Research Foundation, the Grid Laboratory of Wisconsin (GLOW) grid infrastructure at the University of Wisconsin-Madison, the Open Science Grid (OSG) grid infrastructure; U.S. Department of Energy and National Energy Research Scientific Computing Center, the Louisiana Optical Network Initiative (LONI) grid computing resources; Natural Sciences and Engineering Research Council of Canada, WestGrid and Compute/Calcul Canada; Swedish Research Council, Swedish Polar Research Secretariat, Swedish National Infrastructure for Computing (SNIC), and Knut and Alice Wallenberg Foundation, Sweden; German Ministry for Education and Research (BMBF), Deutsche Forschungsgemeinschaft (DFG), Helmholtz Alliance for Astroparticle Physics (HAP), Research Department of Plasmas with Complex Interactions (Bochum), Germany; Fund for Scientific Research (FNRS-FWO), FWO Odysseus programme, Flanders Institute to Encourage Scientific and Technological Research in Industry (IWT), Belgian Federal Science Policy Office (Belspo); University of Oxford, United Kingdom; Marsden Fund, New Zealand; Australian Research Council; Japan Society for Promotion of Science (JSPS); the Swiss National Science Foundation (SNSF), Switzerland; National Research Foundation of Korea (NRF); Danish National Research Foundation, Denmark (DNRF). Some of the results in this paper have been derived using the HEALPix [75] package. We thank R. Laha, J. Beacom, K. Murase, S. Razzaque, and N. Harrington for helpful discussions.

\footnotetext{
*To whom all correspondence should be addressed.

Also at Earthquake Research Institute, University of Tokyo, Bunkyo, Tokyo 113-0032, Japan.

*Also at NASA Goddard Space Flight Center, Greenbelt, Maryland 20771, USA.
} 
[1] S. H. Margolis, D. N. Schramm, and R. Silberberg, Astrophys. J. 221, 990 (1978).

[2] F. W. Stecker, Astrophys. J. 228, 919 (1979).

[3] P. L. Biermann, Cosmic Gamma Rays, Neutrinos, and Related Astrophysics, in Proceedings of the NATO Advanced Study Institute, Erice, Italy, 1989, edited by M. M. Shapiro and J. P. Wefel (Kluwer Academic Publishers, Dordrecht, The Netherlands, 1989), pp. 21-37.

[4] W. Michalak, J. Wdowczyk, and A. W. Wolfendale, J. Phys. G 16, 1917 (1990).

[5] A. Mücke, R. Engel, J. P. Rachen, R. J. Protheroe, and T. Stanev, Comput. Phys. Commun. 124, 290 (2000).

[6] S. Kelner, F. Aharonian, and V. Bugayov Phys. Rev. D 74, 034018 (2006).

[7] R. Abbasi et al. (IceCube Collaboration), Nucl. Instrum. Methods Phys. Res., Sect. A 601, 294 (2009).

[8] M. G. Aartsen et al. (IceCube Collaboration), Phys. Rev. D 89, 102001 (2014).

[9] M. G. Aartsen et al. (IceCube Collaboration), Phys. Rev. D 89, 062007 (2014).

[10] M. G. Aartsen et al. (IceCube Collaboration), Phys. Rev. Lett. 111, 021103 (2013).

[11] M. G. Aartsen et al. (IceCube Collaboration), Science 342, 1242856 (2013).

[12] M. G. Aartsen et al. (IceCube Collaboration), JINST 9, P03009 (2014).

[13] J. F. Beacom and J. Candia, J. Cosmol. Astropart. Phys. 11 (2004) 009.

[14] T. K. Gaisser, S. A. Bludman, H. Lee, and T. Stanev, Phys. Rev. Lett. 51, 223 (1983).

[15] C. Gonzalez-Garcia, M. Maltoni, and J. Rojo, J. High Energy Phys. 10 (2006) 075.

[16] P. Lipari, M. Lusignoli, and D. Meloni, Phys. Rev. D 75, 123005 (2007).

[17] A. Fedynitch, J. Becker Tjus, and P. Desiati, Phys. Rev. D 86, 114024 (2012).

[18] M. Honda, T. Kajita, K. Kasahara, S. Midorikawa, and T. Sanuki, Phys. Rev. D 75, 043006 (2007).

[19] V. S. Berezinsky, D. Cline, and D. N. Schramm, Phys. Lett. 78B, 635 (1978).

[20] L. V. Volkova, Proceedings of the 18th International Cosmic Ray Conference, Bangalore, India, 1983 (Tata Institute of Fundamental Research, Mumbai, 1995), p. 22.

[21] L. V. Volkova, Phys. Lett. B 462, 211 (1999).

[22] G. Gelmini, P. Gondolo, and G. Varieschi, Phys. Rev. D 61, 056011 (2000).

[23] A. D. Martin, M. G. Ryskin, and A. M. Stasto, Acta Phys. Pol. B 34, 3273 (2003).

[24] R. Enberg, M. H. Reno, and I. Sarcevic, Phys. Rev. D 78, 043005 (2008).

[25] M. H. Reno, in American Institute of Physics Conference Series, American Institute of Physics Conference Series Vol. 1560, edited by B. Fleming (2013) pp. 350-354.

[26] T. K. Gaisser, in European Physical Journal Web of Conferences, European Physical Journal Web of Conferences Vol. 52 (2013) p. 9004.

[27] S. Schönert, T. K. Gaisser, E. Resconi, and O. Schulz, Phys. Rev. D 79, 043009 (2009).

[28] T. K. Gaisser, K. Jero, A. Karle, and J. van Santen, arXiv:1405.0525 [Phys. Rev. Lett. (to be published)].
[29] See Supplemental Material at http://link.aps.org/ supplemental/10.1103/PhysRevLett.113.101101 for data tables and event views.

[30] T. K. Gaisser, F. Halzen, and T. Stanev, Phys. Rep. 258, 173 (1995).

[31] J. G. Learned and K. Mannheim, Annu. Rev. Nucl. Part. Sci. 50, 679 (2000).

[32] F. Halzen and D. Hooper, Rep. Prog. Phys. 65, 1025 (2002).

[33] J. K. Becker, Phys. Rep. 458, 173 (2008).

[34] J. Braun, J. Dumm, F. de Palma, C. Finley, A. Karle, and T. Montaruli, Astropart. Phys. 29, 299 (2008).

[35] A. A. Abdo et al. (Milagro Collaboration), Astrophys. J. 688, 1078 (2008).

[36] M. G. Aartsen et al., Astrophys. J. 779, 132 (2013).

[37] S. Adrián-Martínez et al., Astrophys. J. 760, 53 (2012).

[38] S. Adrián-Martínez et al., Astrophys. J. Lett. 786, L5 (2014).

[39] A. D. Supanitsky, Phys. Rev. D 89, 023501 (2014).

[40] M. Ahlers and K. Murase, arXiv:1309.4077.

[41] C. Lunardini, S. Razzaque, K. T. Theodoseau, and L. Yang, arXiv:1311.7188.

[42] Y. Bai, R. Lu, and J. Salvado, arXiv:1311.5864.

[43] A. M. Taylor, S. Gabici, and F. Aharonian, Phys. Rev. D 89, 103003 (2014).

[44] M. Aartsen et al. (IceCube Collaboration), arXiv: 1309.6979.

[45] F. W. Stecker, C. Done, M. H. Salamon, and P. Sommers, Phys. Rev. Lett. 66, 2697 (1991).

[46] O. E. Kalashev, A. Kusenko, and W. Essey, Phys. Rev. Lett. 111, 041103 (2013).

[47] A. M. Atoyan and C. D. Dermer, New Astron. Rev. 48, 381 (2004).

[48] J. Alvarez-Muñiz and P. Mészáros, Phys. Rev. D 70, 123001 (2004).

[49] L. Nellen, K. Mannheim, and P. L. Biermann, Phys. Rev. D 47, 5270 (1993).

[50] K. Mannheim, Astropart. Phys. 3, 295 (1995).

[51] F. W. Stecker and M. H. Salamon, Space Sci. Rev. 75, 341 (1996).

[52] J. P. Rachen and P. Mészáros, Phys. Rev. D 58, 123005 (1998).

[53] P. Mészáros and E. Waxman, Phys. Rev. Lett. 87, 171102 (2001).

[54] A. Mücke, R. J. Protheroe, R. Engel, J. P. Rachen, and T. Stanev, Astropart. Phys. 18, 593 (2003).

[55] J. K. Becker, P. L. Biermann, and W. Rhode, Astropart. Phys. 23, 355 (2005).

[56] F. W. Stecker, Phys. Rev. D 72, 107301 (2005).

[57] B. Eichmann, R. Schlickeiser, and W. Rhode, Astrophys. J. 749, 155 (2012).

[58] E. Waxman and J. Bahcall, Phys. Rev. Lett. 78, 2292 (1997).

[59] D. Guetta, D. Hooper, J. Alvarez-Muñiz, F. Halzen, and E. Reuveni, Astropart. Phys. 20, 429 (2004).

[60] P. Baerwald, M. Bustamante, and W. Winter, arXiv: 1401.1820.

[61] W. Winter, J. Becker Tjus, and S. R. Klein, arXiv: 1403.0574.

[62] E. Waxman and J. N. Bahcall, Astrophys. J. 541, 707 (2000). 
[63] S. Razzaque, P. Mészáros, and E. Waxman, Phys. Rev. D 68, 083001 (2003).

[64] J. K. Becker, M. Stamatikos, F. Halzen, and W. Rhode, Astropart. Phys. 25, 118 (2006).

[65] K. Murase and S. Nagataki, Phys. Rev. Lett. 97, 051101 (2006).

[66] A. Loeb and E. Waxman, J. Cosmol. Astropart. Phys. 05 (2006) 003.

[67] K. Murase, M. Ahlers, and B. C. Lacki, Phys. Rev. D 88, 121301 (2013).

[68] T. M. Yoast-Hull, J. S. Gallagher III, E. G. Zweibel, and J. E. Everett, Astrophys. J. 780, 137 (2014).

[69] T. A. Thompson, E. Quataert, E. Waxman, and A. Loeb, Astrophys. J. 654, 219 (2007).
[70] R. Abbasi et al. (IceCube Collaboration), Nature (London) 484, 351 (2012).

[71] E. Waxman and J. Bahcall, Phys. Rev. D 59, 023002 (1998).

[72] K. Mannheim, R. J. Protheroe, and J. P. Rachen, Phys. Rev. D 63, 023003 (2000).

[73] P. Bagley et al., KM3NeT Technical Design Report for a Deep-Sea Research Infrastructure Incorporating a Very Large Volume Neutrino Telescope (KM3NeT Consortium, 2011), http://km3net.org/TDR/TDRKM3NeT.pdf.

[74] R. Laha, J. F. Beacom, B. Dasgupta, S. Horiuchi, and K. Murase, Phys. Rev. D 88, 043009 (2013).

[75] K. M. Górski, E. Hivon, A. J. Banday, B. D. Wandelt, F. K. Hansen, M. Reinecke, and M. Bartelmann, Astrophys. J. 622, 759 (2005). 